\title{
Epigenetics of multiple sclerosis
}

\section{Carleigh Robertson}

Department of Molecular and Cellular Biology, College of Biological Science, University of Guelph, Guelph, ON Canada. Faculty supervisor: Dr. George Harauz. For correspondence, please email: carleigh.robertson@gmail.com or gharauz@uoguelph.ca.

\section{Abstract}

Multiple sclerosis (MS) is a complex disease characterized by autoimmune demyelination of the myelin sheath of the central nervous system. Varying degrees of disability result from disruptions in neural, physical, and psychological processes. No clear cause of MS has been identified, but specific genetic mutations and environmental effects have been established as factors in disease development. However, the low disease concordance rates and transmission disequilibrium observed in MS prevent genetic factors alone from explaining increased susceptibility. As a result, the involvement of epigenetics, or heritable changes in the genome that are not caused by alterations in the underlying DNA sequence, has been proposed to resolve these apparent discrepancies. This article focuses on recent research into three primary epigenetic mechanisms and their relation to MS susceptibility: DNA methylation, histone modifications, and posttranscriptional regulation of target genes by microRNA. These epigenetic phenomena interact with each other and environmental stimuli to produce disease. Promising experimental research has revealed that inhibitors of such epigenetic activity reduce disease phenotypes in animal models. In this article, ways in which epigenetic changes can induce MS development are described and prospective research avenues are proposed.

Keywords: epigenetics; multiple sclerosis (MS); DNA methylation; histones; microRNA (miRNA)

\section{Introduction}

Multiple sclerosis (MS) is the most common demyelinating disease of the central nervous system, affecting 2.0 to 2.5 million people worldwide (Milo and Kahana 2010; Nakahara et al. 2012). MS defies traditional classification, possessing both autoimmune and neurological attributes, and is characterized by the formation of demyelinated foci in the central nervous system and by a variety of chronic physical, neurological, and psychological symptoms (Koch et al. 2013; Zhou et al. 2014).

Demyelination, or damage to the myelin sheath that surrounds axons of the brain and spinal cord, is the result of focal infiltration by lymphocytes, macrophages and antibodies, which results in transient inflammation (IMSGC 2007; Liu et al. 2010). Attempts at remyelination do occur, but often the repair of these lesions is not permanent and subsequent remyelination attempts are increasingly unsuccessful (Compston and Coles 2008). The physical and neurological disabilities that characterize MS are the result of the accumulation of damage over time (Tullman 2013).

MS has consistently been observed in a greater proportion of the population in geographical regions far from the equator. Canada in particular has one of the highest rates of MS worldwide, along with a number of nations in northern Europe (Willer et al. 2005). Charles Poser (1994) suggested that perhaps MS was propagated genetically through Viking lineages, as MS occurs with such high frequency in Scandinavian countries. Much evidence supports this concept of MS susceptibility, with factors such as climate, month of birth, and sun exposure acting as environmental stimuli that influence disease risk and progression through mediation by genetic and epigenetic mechanisms (Koch et al. 2013; Zhou et al. 2014). Other factors, such as smoking and Epstein-Barr virus infection, contribute further to affect an individual's susceptibility to MS (Nischwitz et al. 2011).

\section{Epigenetics of Disease}

\section{Overview}

Changes in the DNA sequence of MS-related genes are only a minor factor in the development of the disease. Emerging data suggest that epigenetics, or changes in gene expression that are not a result of changes in the underlying DNA sequence, may have a greater influence on the development and progression of MS than mutations in any particular locus (Berger et al. 2009; Koch et al. 2013; Zhou et al. 2014). Epigenetic marks are maintained through generations of somatic cells within the body, as well as in gametes, and can therefore be passed from parents to children (Jaenisch and Bird 2003; Berger et al. 2009). However, because of their high sensitivity to environmental stimuli, epigenetic marks are uniquely capable of being altered or reversed over time (Jaenisch and Bird 2003). Three primary epigenetically-regulated mechanisms play key roles 
in the pathophysiology of MS: DNA methylation, histone modifications, and microRNA-associated post-transcriptional gene regulation.

\section{DNA methylation}

DNA methylation is currently the most widely studied epigenetic mechanism, and involves the addition of a methyl group to cytosine residues in $\mathrm{CpG}$ dinucleotides within a DNA sequence via DNA methyltransferase (DNMT) activity (Liu et al. 2010). Hypermethylated cytosine residues within a promoter region interfere with sequence recognition by transcription factors. As a result, this type of modification is usually associated with the stable repression of gene expression at the associated locus (Klose and Bird 2006; Huynh and Casaccia 2013).

\section{Histone modifications}

Genes may also be regulated epigenetically through modifications to the superstructure of DNA. One such component of this condensed structure are histones, protein octamers around which DNA is coiled (Dieker and Muller 2010). Gene expression can be modulated by various posttranslational modifications, including methylation or acetylation, and the combined effects of these modifications confer a unique histone code to distinct cell types (Copray et al. 2009; Huynh and Casaccia 2010). The general mechanism by which gene expression is altered is through changes in chromatin structure that affect the accessibility of specific genetic loci to transcription machinery (Copray et al. 2009; Koch et al. 2013). Whether gene expression is repressed or activated depends on the particular combination of modifications that are found on histones, which will either condense the chromatin to repress expression or expand it to enhance transcription (Alva et al. 2007).

\section{miRNA-associated post-transcriptional gene resultation}

MicroRNAs (miRNAs) are small single-stranded, noncoding molecules formed from larger transcripts that fold to produce hairpin structures (Sevignani et al. 2006). After several nuclear and cytoplasmic processing steps, miRNAs complex with a host of proteins to produce the RNA-induced silencing complex (RISC) that enables miRNA to regulate gene expression by binding to associated mRNA transcripts (Bartel 2004). Imperfect base pairing causes the target mRNA to be degraded, whereas perfect base pairing induces translational repression of the target's gene product through the inhibition of translation (Bartel 2004).

\section{The Role of Epigenetics in MS}

The clinical attributes of MS suggest that underlying epigenetic mechanisms may contribute to disease susceptibility and progression. For instance, the low concordance rate of MS in twins (roughly 5\% in dizygotic twins and 25-30\% in monozygotic twins) implies that genetic polymorphisms alone cannot be the cause of the disease since identical twins share $100 \%$ of their genes (Compston and Coles 2008; Ramagopalan and Ebers 2010). In addition, epigenetic mechanisms are thought to mediate genomic imprinting that may account for the increased prevalence of $\mathrm{MS}$ in women. For instance, the $D R B 1 * 15$ allele of the $H L A$ gene, which is involved in immunity and is associated with an elevated MS risk, is carried by more women than men (Chao et al. 2010).

Although the epigenetic mechanisms described here have their own distinct effects, it is important to note that these processes interact rather than act in isolation. Cancer studies suggest that DNA methylation and histone modifications are capable of modulating miRNA expression, which themselves are regulated by other miRNAs (Han et al. 2007; Koch et al. 2013). Other interactions occur between methylated DNA and histone-modifying enzymes, such as recruitment of DNMTs by histones to induce de novo DNA methylation (Zhao et al. 2009). These basic epigenetic mechanisms also exert their effects in concert with specific environmental factors (Koch et al. 2013; Zhou et al. 2014).

Taken together, various aspects of MS pathophysiology and disease development may be produced as a result of epigenetic changes that induce effects of their own, while also interacting in complex networks that are not yet completely understood.

\section{DNA methylation and MS pathogenesis}

Methylation of $\mathrm{CpG}$ dinucleotides in a number of gene promoter regions that may be responsible for the immune properties of MS have been described. The immunopathology of the disease involves the dominance of $\mathrm{T}$ helper cell $1\left(\mathrm{~T}_{\mathrm{H}} 1\right)$ immunity, associated with the cytokine interferon- $\gamma$ (IFN- $\gamma$ ), over $\mathrm{T}_{\mathrm{H}} 2$ cell immunity (Kürtüncü and Tüzün 2008). The differentiation of the two $\mathrm{T}$ cell types is regulated by epigenetic mechanisms, as is the production of IFN- $\gamma$ and other cytokines (Kürtüncü and Tüzün 2008). Aberrant patterns of DNA methylation have been observed in the promoter region for IFN- $\gamma$ within $\mathrm{T}$ helper cells, which may be related to the dominance of $T_{H} 1$ immunity over $T_{H} 2$ immunity in MS (Kürtüncü and Tüzün 2008).

Analyses of brain tissue samples from MS patients have identified an association between DNA methylation and neuronal cell death (Chestnut et al. 2011). In this study, overexpression of the enzyme responsible for de novo methylation of DNA induced apoptosis in cells. Furthermore, expression of other DNMTs increased when apoptosis was induced.

Smoking is known to be a risk factor for developing MS and is associated with more frequent relapses and more numerous MS lesions in patients (Ascherio and Munger 2007). Studies on the link between cigarette smoking and cancer have determined that smoking induces the silencing of tumour suppressor genes, mostly through DNA hypermethylation (Wan et al. 2012).

Hypermethylation of the promoter regions of tumour suppression genes is also associated with Epstein-Barr virus (EBV) infection (Niller et al. 2009). Studies involving cancer 
have demonstrated that the viral latent membrane protein 1 (LMP1) acts to up-regulate various DNMTs, which in turn induce hypermethylation at various promoter sites (Tsai et al. 2002). In fact, individuals with a history of infective mononucleosis, a type of EBV infection, double their risk of developing MS because of changes in the epigenetic profile of infected cells (Handel et al. 2010).

Hypomethylation of some promoters can also have an effect on MS development. For instance, when the promoter of the PAD2 gene is hypomethylated, the enzyme PAD2 is overexpressed (Mastronardi et al. 2007). This enzyme is responsible for the citrullination of myelin basic protein (MBP), which is a key player in MS pathophysiology. The modified form of MBP is less stable, and the conversion of positively-charged arginyl residues to neutral citrullinyl residues can result in less compact myelin that is prone to disintegration and a possible autoimmune reaction to MBP (Musse and Harauz 2007; Mastronardi et al. 2007). Brain biopsy samples from MS patients demonstrated white matter that appeared normal, but contained increased levels of citrullinated MBP in comparison to samples from healthy control children, adults, and individuals with other neurodegenerative diseases, such as Alzheimer's, Huntington's or Parkinson's (Moscarello et al. 1994).

\section{Histone modifications and MS pathogenesis}

A number of modifications to histones have been found to be associated with the development and manifestation of MS. In a study of patients with progressive MS and controls without neurological disease, an increase in histone $\mathrm{H} 3$ acetylation (the addition of acetyl groups) in neural cells known as oligodendrocytes within chronic MS lesions was found, whereas early-stage MS lesions contained oligodendrocytes with marked histone deacetylation (the removal of acetyl groups) (Pedre et al. 2011). The researchers noted that in rodent models, histone deacetylation appeared to favour differentiation of oligodendrocytes, whereas acetylation resulted in impaired differentiation because of increased expression of transcriptional inhibitors of cell differentiation (Pedre et al. 2011). Reduced oligodendrocyte differentiation might lead to impaired remyelination, providing a potential link to lesion development (Koch et al. 2013). Furthermore, the study demonstrated that histone $\mathrm{H} 3$ acetylation is increased in samples from older patients and the extent of acetylation is correlated with the severity of disease (Pedre et al. 2011).

There is a significant impact of histone structure on oligodendrocyte development. Histone deacetylases (HDACs: enzymes that remove acetyl groups from histone tails) inhibit development into alternative cell lineages and repress myelin-specific gene inhibitors in oligodendrocyte progenitor cells (Shen and Casaccia-Bonnefil 2008). HDAC1 is recruited to the proximal promoter region of MBP genes by transcription factors Nkx2.2 and Hes5 (Wei et al. 2005). These changes in gene expression mediate oligodendrocyte maturation, a process that is often aberrant in MS and which leads to impaired remyelination (Copray et al. 2009).
HDAC inhibitors have been shown to have positive effects in animal models of MS. Camelo et al. found in 2005 that administering trichostatin A (TSA) to mice with experimental autoimmune encephalomyelitis (EAE), an animal model of MS, attenuated spinal cord inflammation, demyelination and axonal loss. Interestingly, Natajaran and Bright (2002) reported that curcumin, a selective histone acetyltransferase inhibitor, which prevents acetylation of histones, also prevented EAE from developing in mice, and blocked the IL-12 signalling pathway in T cells. No similar studies have been undertaken in humans, but these observations lend support to the role of histone modifications in MS and other demyelinating disorders.

Biochemical alterations to histones play a role in the increased risk that vitamin D deficiency confers to MS. In fact, epidemiological studies suggest that as blood levels of 25-hydroxyvitamin $\mathrm{D}_{3}$ increase, the risk of developing MS decreases (Munger et al. 2006). Individuals with relapseremitting MS tend to experience greater disability and more frequent relapses with reduced levels of vitamin $\mathrm{D}$, which is complicated by the fact that they may experience difficulty in obtaining sun exposure, a major factor in vitamin D production, due to physical disability (Smolders et al. 2008). The manner in which vitamin D exerts an effect on MS susceptibility and severity is unclear, but studies on vitamin D deficiency and cancer suggest that it may epigenetically influence disease by interacting with histones: 25hydroxyvitamin $\mathrm{D}$ induces the expression of JMJD3, a gene that encodes histone $\mathrm{H} 3$ demethylase, an enzyme that removes methyl groups from histones, and thereby alters the overall chromatin structure of target genes (Pereira et al. 2011). Vitamin D is also associated with strong immunemediating functions, and is even capable of preventing the onset and development of EAE in mice (Cantorna et al. 1996). Mice that were administered vitamin $D$ at the onset of neurological symptoms, then later had this treatment discontinued, resumed EAE progression (Cantorna et al. 1996).

The influence of vitamin D on MS is also implicated in the increased prevalence of MS in individuals born in the spring than those born in winter months, as concluded by Willer and colleagues in their evaluation of MS patients in Canada, along with similar data pooled from Great Britain, Denmark, Sweden, and other nations (Willer et al. 2005). These investigations indicated that a significantly higher number of patients with MS were born in May than those born in November, and that this variation may be related to the higher geographical latitude of the countries studied and thus the level of sun exposure in those regions (Willer et al. 2005). It has not been conclusively established why these patterns of increased MS risk have been observed, and further investigation into these findings could be an interesting and worthwhile area of future research.

Finally, histone citrullination, which is the enzymatic conversion of arginine residues to citrulline residues, has a significant impact on the etiopathogenesis of MS as well. In the presence of augmented levels of tumour necrosis factor $\alpha$ 
(TNF $\alpha$ ), the enzyme PAD4, which is related to PAD2, relocates from the cytoplasm to the nucleus and increases histone citrullination (Mastronardi et al. 2006; Copray et al. 2009). Mastronardi et al. (2006) found that citrullination occurs prior to the onset of symptoms in mice with EAE and observed an increase in histone citrullination in the white matter of MS patients in comparison to control subjects.

\section{miRNA-associated post-transcriptional regulation in MS}

The bulk of our current understanding of how MS is regulated by epigenetic mechanisms is derived from studies that focus on patients with actively-demyelinating lesions. In one study, distinct miRNA profiles were found in biopsy samples from active MS lesions from affected individuals that showed significant up-regulation of $m i R-155, m i R-34 \mathrm{a}$, and miR-326 (Junker et al. 2009). The miRNA transcripts of this family of genes target CD47, a cell surface protein that inhibits the phagocytic activity of macrophages and is widely expressed in cells (Junker et al. 2009). The miRNAs decrease CD47 expression, which leads to the activation of macrophages and subsequently, the phagocytic destruction of myelin, thus producing an active lesion if the cell is unable to induce remyelination (Chari 2007; Junker et al. 2009).

Another group of studies found that $m i R-155$ is also associated with $\mathrm{T}$ cell differentiation, with $m i R-326$ performing similar functions (O'Connell et al. 2010). Upregulation of miR-155 in some types of leukocytes (macrophages, B cells, and $\mathrm{T}$ cells), in response to ligand binding to toll-like receptors and inflammatory cytokines, suggests that miRNA may be involved in an inflammatory response (O'Connell et al. 2010). It has also been found that mice deficient in $m i R-155$ are resistant to developing EAE when compared to wild-type mice (O'Connell et al. 2010). In addition, a study of MS patients and mice with EAE found that $m i R-326$ was highly up-regulated in peripheral blood cells, and this increase in expression was most pronounced during patient relapses, indicating that miR-326 is highly correlated with disease severity (Du et al. 2009). In contrast, control subjects and MS patients in remission exhibited lower levels of $m i R-326$ expression (Du et al. 2009). $m i R$ 326 targets a transcription factor that inhibits $\mathrm{T}$ cell differentiation; therefore, overexpression of $m i R-326$ suppresses the expression of this protein and subsequently increases production of certain kinds of $\mathrm{T}$ cells (Du et al. 2009). This event can lead to the immune changes associated with MS development.

Up-regulation of other miRNA genes has been implicated in MS. In addition to $m i R-155$, increased expression of $m i R-338$ and $m i R-491$ in brain samples, obtained from patients with progressive MS, caused the transcribed miRNAs to target and suppress the translation of enzymes in the aldo-keto reductase family, which are involved in the production of neurosteroids, such as allopregnalone, in brain white matter (Noorbakhsh et al. 2011). Researchers found that in mice with EAE, overexpression of $m i R-338$ and $m i R-491$ decreased the production of allopregnalone, whereas mice that were administered the neurosteroid exhibited reduced brain inflammation and attenuated myelin and axonal damage, suggesting that allopregnalone possesses neuroprotective properties (Noorbakhsh et al. 2011). Endogenous neurosteroid synthesis is regulated by miRNA-associated post-transcriptional silencing, and as such, the dysregulation of synthesis is an important pathological mechanism in progressive MS (Noorbakhsh et al. 2011).

\section{Future Research Related to the Epigenetics of MS}

As epigenetics is a relatively new area of research, most studies of the epigenetic mechanisms involved in MS are preliminary, but open doors for future studies that can extrapolate from the ideas they present and explore potential applications in greater detail. They may also serve to clarify discrepancies between findings in studies of similar phenomena: for example, not all evidence has supported DNA methylation as a significant factor in MS development. Baranzini et al. (2010) undertook research assessing genetic, epigenetic, and transcriptomic differences in CD4+ T helper cells between three monozygotic twin pairs, in which one twin suffered from MS, in an attempt to explain disease discordance. However, no relevant differences in DNA methylation patterns were found in the genomes of $\mathrm{T}$ cells in co-twins, providing little support for explaining discordance, and suggesting that other epigenetic mechanisms may play a role in MS pathogenesis (Baranzini et al. 2010). In the same year, Ramagopalan and Ebers (2010) suggested that because somatic cells accumulate and alter their epigenetic marks over the course of an individual's lifetime in response to their environment, monozygotic twins may not, in fact, have identical gene expression, and therefore might have substantial differences in their epigenome. They state, however, that although the Baranzini et al. study appears to tentatively rule out epigenetic changes as a factor in MS development, other cell types should be examined and the genomes of more than a few twin pairs should be sequenced in order to make more accurate judgments about the influence of epigenetics in disease development (Ramagopalan and Ebers 2010). Furthermore, as the costs of sequencing studies, such as the one undertaken by Baranzini et al., continue to decrease, new revelations may provide stepping stones for important future research directions.

Other general recommendations can be made for prospective studies in MS epigenetics. Larger study populations should be employed in research involving both general MS patients and twins. Studies on twins and disease concordance rates that have been published thus far have used very small cohorts of only a few twin pairs, and thus may not represent a thorough understanding of how epigenetics in MS is involved in concordance and heredity. Furthermore, existing studies, especially recent publications, 
should be replicated to validate current findings, preferably with larger cohorts.

Of particular interest is research that links neuroimaging with epigenetic findings. Magnetic resonance imaging (MRI) and positron emission tomography (PET) are especially useful. Studies could be designed that compare specific epigenetic marks found in MS patients with lesion progression over time in brain matter or with the type of MS exhibited by the patient.

Similarly, longitudinal studies of MS patients and the epigenetic marks they manifest would be beneficial. Researchers could examine changes in DNA methylation, functional groups attached to histone tails, or miRNA expression at multiple intervals throughout an individual's lifetime, and observe whether there are differences over time. Are the epigenetic marks stable? Do they correlate with disease severity or periods of remission? Families with multiple affected members are also useful study subjects as they provide the opportunity to investigate whether epigenetic marks related to MS development are propagated stably from parents to their children.

Some studies have been completed that investigate the use of DNA methylation as a biomarker for disease activity in cancer (Feinberg and Tycko, 2004; Liggett et al. 2010). Extending those applications to MS could provide a reliable means of diagnosing the disease, and perhaps in predicting the course of relapse and remission. Sodium bisulphite mapping is a useful experimental tool for determining the specific $\mathrm{CpG}$ islands within a gene that are methylated. Studies involving this technique may be used to compile a library of methylation sites that are of greatest significance in MS patients.

Upregulation of DNMTs in apoptosis could be involved in the neuronal degeneration seen in MS, but no studies have examined their upregulation as a possible disease mechanism. It may be prudent to begin this investigation in future studies of EAE in animal models, likely mice, with EAE as a representation of MS.

In 2004, Mastronardi et al. proposed an experimental therapeutic strategy that epigenetically reprograms oligodendrocytes by favouring methylation at the promoter of IFN- $\gamma$ genes. INF- $\beta$ is currently a standard treatment in relapse-remitting MS for decreasing the number of symptomatic episodes, but combinatorial therapy of IFN- $\beta$ with vitamin B12 down-regulated IFN- $\gamma$ expression in in vitro studies of oligodendrocytes (Mastronardi et al. 2004). IFN- $\beta$ alone increased the number of processes in developing oligodendrocytes, but combinatorial treatment significantly increased oligodendrocyte maturation, as well as attenuated inflammation (Mastronardi et al. 2004). Because in vitro experiments produced positive results in oligodendrocyte maturation, in vivo studies involving this combinatorial therapy are possible next steps.

The study conducted by Pedre et al. (2011) suggested that histone acetylation and other histone modifications may have a role in the pathogenesis and physiology of MS, but that further research is necessary in order to increase our understanding of the effects of these changes to histones. The ultimate functional effects of histone modifications should be examined as well.

A great deal of research has begun on the roles that miRNA play in the development of MS by interacting with the genome, and future research may uncover other miRNAs that affect MS pathogenesis. It may be useful to inquire as to whether transcription factors or other small molecules also control gene expression in the disease. Since the precise functions of each miRNA may be difficult to determine in humans because of the multitude of other factors that contribute to MS pathophysiology, using specific cell lines or animal models may be more feasible.

There are other questions that remain that relate to MS susceptibility and disease progression. Although it has been widely acknowledged that EBV infection increases an individual's MS risk, it remains to be demonstrated exactly how this elevated risk is generated. Is the increase related to the changes in the epigenetic profile of infected cells as suggested by Handel and colleagues (2010)? What types of epigenetic changes in these cells are correlated with MS? More research is required to determine the role of EBV in MS pathogenesis and how it evokes autoimmunity in affected individuals.

MS researchers continue to make strides in further elucidating disease mechanisms and developing novel treatments, innovations that will undoubtedly contribute to our collective knowledge of MS and the ultimate wellbeing of MS sufferers. The study of epigenetics in MS is expanding significantly our perspective on the complex factors that interact to produce the disease.

\section{Acknowledgements}

The author wishes to thank Dr. George Harauz for supervising this project, assisting with the research, and reviewing the manuscript. The work in Dr. Harauz's laboratory is supported by the Natural Sciences and Engineering Research Council (NSERC) of Canada, the Canada Research Chairs Program, and the Ontario-Manitoba endMS Regional Research and Training Network of the Multiple Sclerosis Society of Canada.

\section{References}

Alva V, Ammelburg M, Soding J, Lupas A. 2007. On the origin of the histone fold. BMC Structural Biology. 7:17.

Ascherio A, Munger KL. 2007. Environmental risk factors for multiple sclerosis. Part II: noninfectious factors. Annals of Neurology. 61:504-513.

Baranzini SE, Mudge J, van Velkinburgh JC, Khankhanian P, Khrebtukova I, Miller NA, Zhang L, Farmer AD, Bell CJ, Kim RW, et al. 2010. Genome, epigenome and RNA sequences of monozygotic twins discordant for multiple sclerosis. Nature. 464:1351-1356. 
Bartel DP. 2004. MicroRNAs: genomics, biogenesis, mechanism and function. Cell. 116:281-297.

Berger SL, Kouzarides T, Shiekhattar R, Shilatifard S. 2009. An operational definition of epigenetics. Genes and Development. 23:781-783.

Camelo S, Iglesias AH, Hwang D, Due B, Ryu H, Smith K, Gray SG, Imitola J, Duran G, Assaf B et al. 2005. Transcriptional therapy with the histone deacetylase inhibitor trichostatin A ameliorates experimental autoimmune encephalomyelitis. Journal of Immunology. 164(1-2):10-21.

Cantorna MT, Hayes CE, Deluca HF. 1996. 1,25dihydroxyvitamin $\mathrm{D}_{3}$ reversibly blocks the progression of relapsing encephalomyelitis, a model of multiple sclerosis. Proceedings of the National Academy of Sciences. 93:7861-7864.

Chao M.J, Herrera BM, Ramagopalan SV, Deluca G, Handunetthi L, Orton SM, Lincoln MR, Sadovnick AD, Ebers GE. 2010. Parent-of-origin effects at the major histocompatibility complex in multiple sclerosis. Human Molecular Genetics. 19:3679-3689.

Chari D. 2007. Remyelination in multiple sclerosis. International Review of Neurobiology. 79:589-620.

Chestnut BA, Chang Q, Price A, Lesuisse C, Wong M, Martin LJ. 2011. Epigenetic regulation of motor neuron cell death through DNA methylation. Journal of Neuroscience. 46:16619-16636.

Compston A, Coles A. 2002. Multiple sclerosis. The Lancet. 359:1221-1231.

Compston A, Coles A. 2008. Multiple sclerosis. The Lancet. 372:1502-1517.

Copray S, Huynh JL, Sher F, Casaccia-Bonnefil P, Boddeke E. 2009. Epigenetic mechanisms facilitating oligodendrocyte development, maturation and aging. Glia. 57:5179-1587.

Dieker J, Muller S. 2010. Epigenetic histone code and autoimmunity. Clinical Reviews in Allergy and Immunology. 39:78-84.

Du C, Liu C, Kang J, Zhao G, Ye Z, Huang S, Li Z, Wu Z, Pei G. 2009. MicroRNA miR-326 regulates $T_{\mathrm{H}^{-17}}$ differentiation and is associated with the pathogenesis of multiple sclerosis. Nature Immunology. 10:1252-1259.

Ebers GC, Yee IM, Sadovnick AD, Duquette P. 2000. Conjugal multiple sclerosis: population-based prevalence and recurrence risks in offspring. Annals of Neurology. 48:927-931.

Ebers GC, Sadovnick AD, Dyment DA, Yee IM, Willer CJ, Risch N. 2004. Parent-of-origin effect in multiple sclerosis: observations in half-siblings. The Lancet. 363:1773-1774.
Feinberg AP, Tycko B. 2004. The history of cancer epigenetics. Nature Reviews Cancer. 4:143-153.

Han L, Witmer PD, Casey E, Valle D, Sukumar S. 2007. DNA methylation regulates microRNA expression. Cancer Biology and Therapy. 6:1284-1288.

Hande, AE, Williamson AJ, Disanto G, Handunnetthi L, Giovannoni G, Ramagopalan SV. 2010. An updated meta-analysis of risk of multiple sclerosis following infectious mononucleosis. Public Library of Science (PLoS) ONE. 5:e12496.

Hernán MA, Jick SS, Logroscino G, Olek, MJ, Ascherio A, Jick H. 2005. Cigarette smoking and the progression of multiple sclerosis. Brain. 128:1461-1465.

Hoppenbrouwers IA, Aulchenko YS, Janssens AC, Ramagopalan SV, Broer L, Kayser M, Ebers GC, Oostra BA, van Duijn CM, Hintzen RQ. 2009. Replication of CD58 and CLEC16A as genome-wide significant risk genes for multiple sclerosis. Journal of Human Genetics. 54:676-680.

Huynh JL, Casaccia P. 2010. Defining the chromatin landscape in demyelinating disorders. Neurobiology of Disease. 39:47-52.

Huynh JL, Casaccia P. 2013. Epigenetic mechanisms in multiple sclerosis: implications for pathogenesis and treatment. The Lancet Neurology. 12:195-206.

[IMSGC] International Multiple Sclerosis Genetics Consortium. 2007. Risk alleles for multiple sclerosis identified by a genome-wide study. New England Journal of Medicine. 357: 851-862.

[IMSGC] International Multiple Sclerosis Genetics Consortium. 2008. Refining genetics associations in multiple sclerosis. The Lancet Neurology. 7:567-569.

Jaenisch R, Bird A. 2003. Epigenetic regulation of gene expression: how the genome integrates intrinsic and environmental stimuli. Nature Genetics. 33:245-254.

Junker A, Krumbholz M, Eisele S, Mohan H, Augstein F, Bittner R, Lassmann H, Werkele H, Hohlfeld R, Meinl E. 2009. MicroRNA profiling of multiple sclerosis lesions identifies modulators of the regulatory protein CD47. Brain. 132:3342-3352.

Koch MW, Metz LM, Kovalchuk O. 2013. Epigenetics and miRNAs in the diagnosis and treatment of multiple sclerosis. Trends in Molecular Medicine. 19:23-30.

Koch MW, Metz LM, Kovalchuk O. 2013. Epigenetic changes in patients with multiple sclerosis. Nature Reviews Neurology. 9:35-43.

Klose RJ, Bird A. 2006. Genomic DNA methylation: the mark and its mediators. Trends in Biochemical Sciences. 31:89-97. 
Kürtüncü M, Tüzün E. 2008. Multiple sclerosis: could it be an epigenetic disease? Medical Hypotheses. 71:945-947.

Liggett T, Melnikov A, Tilwalli S, Yi Q, Chen H, Replogle C, Feng X, Reder A, Stefoski D, Balabanov R, et al. 2010. Methylation patterns of cell-free plasma DNA in relapsing-remitting multiple sclerosis. Journal of Neurological Science. 290:16-21

Liu J, Sandoval J, Doh ST, Cai L, Lopez-Rodas G, Cassacia P. 2010. Epigenetic modifiers are necessary but not sufficient for reprogramming non-myelinating cells into myelin gene-expressing cells. Public Library of Science (PLoS) ONE. 5: e13023.

Mastronardi FG, Wood DD, Mei J, Raijmakers R, Tseveleki V, Dosch HM, Probert L, Cassacia-Bonnefil P, Moscarello MA. 2006. Increased citrullination of histone H3 in multiple sclerosis brain and animal models of demyelination: a role for tumour necrosis factor-induced peptidylarginine deiminase 4 translocation. Journal of Neuroscience. 26:11387-11396.

Mastronardi FG, Noor A, Wood DD, Paton T, Moscarello MA. 2007. Peptidylarginine deiminase $2 \mathrm{CpG}$ island in multiple sclerosis white matter is hypomethylated. Journal of Neuroscience Research. 85:2006-2016.

Milo R, Kahana E. 2010. Multiple sclerosis: geoepidemiology, genetics and the environment. Autoimmunity Reviews. 9:387-394.

Moscarello MA, Wood DD, Ackerly C, Boulias C. 1994. Myelin in multiple sclerosis is developmentally immature. Journal of Clinical Investigation. 94:146-154.

Munger KL., Levin LI, Hollis BW, Howard NS, Ascherio A. 2006. Serum 25-hydroxyvitamin D levels and risk of multiple sclerosis. Journal of the American Medical Association. 296:2832-2838.

Musse AA, Harauz G. 2007 Molecular 'negativity' may underlie multiple sclerosis (MS): role of the myelin basic protein family in the pathogenesis of MS. International Review of Neurobiology. 79C: 149-172.

Nakahara J, Maeda M, Aiso S, Suzuki N. 2012. Current concepts in multiple sclerosis: autoimmunity versus oligodendrogliopathy. Clinical Reviews in Allergy and Immunology. 42:26-34.

Natajaran C, Bright JJ. 2002. Curcumin inhibits experimental autoimmune encephalomyelitis by blocking IL-12 signalling through Janus kinase-STAT pathway in T lymphocytes. Journal of Immunology. 168: 6506-6513.

Niller HH, Wolf H, Minarovits J. 2009. Epigenetic dysregulation of the host cell genome in Epstein-Barr virus-associated neoplasia. Seminars in Cancer Biology. 19:158-164.
Nischwitz S, Müller-Myhsok B, Weber F. 2011. Risk conferring genes in multiple sclerosis. FEBS Letters. 585:3789-3797.

Noorbakhsh F, Ellestad KK, Maingat F, Warren KG, Han MH, Steinman L, Baker GB, Power C. 2011. Impaired neurosteroid synthesis in multiple sclerosis. Brain. 134: 2703-2721.

O’Connell RM, Kahn D, Gibson WSJ, Round JL, Scholz RL, Chaudhuri AA, Kahn ME, Rao DS, Baltimore D. 2010. MicroRNA-155 promotes autoimmune inflammation by enhancing inflammatory $\mathrm{T}$ cell development. Immunity. 33: 607-619.

Oksenberg JR, Barcellos LF. 2005. Multiple sclerosis genetics: leaving no stone unturned. Genes and Immunity. 6:375-387.

Pedre X, Mastronardi F, Bruck W, Lopez-Rodas G, Kuhlmann T, Casaccia P. 2011. Changed histone acetylation patterns in normal-appearing white matter and early multiple sclerotic lesions. Journal of Neuroscience. 31:3435-3445.

Pereira F, Barbachano A, Silva J, Bonilla F, Campbell MJ, Munoz A, Larriba MJ. 2011. KDM6B/JMJD3 histone demethylase is induced by vitamin $\mathrm{D}$ and modulates its effects in colon cancer cells. Human Molecular Genetics. 20: 4655-4665.

Poser CM. 1994. The dissemination of multiple sclerosis: a Viking saga? A historical essay. Annals of Neurology. 36:231-234.

Ramagopalan SV, Ebers GC. 2010. Sequencing the multiple sclerosis genome: more questions than answers. The Lancet Neurology. 9:757-759.

Sadovnick AD. 2012. Genetic background of multiple sclerosis. Autoimmunity Reviews. 11:163-166.

Sevignani C, Calin GA, Siracusa LD, Croce CM. 2006. Mammalian microRNAs: a small world for fine-tuning gene expression. Mammalian Genome. 17:189-202.

Shen S, Casaccia-Bonnefil P. 2008. Post-translational modifications of nucleosomal histones in oligodendrocyte lineage cells in development and disease. Journal of Molecular Neuroscience. 35:13-22.

Smolders J, Menheere P, Kessels A, Damoiseaux J, Hupperts R. 2008. Association of vitamin D metabolite levels with relapse rate and disability in multiple sclerosis. Multiple Sclerosis Journal. 14:1220-1224.

Tsai CN, Tsa, CL, Tse KP, Chang HY, Chang YS. 2002. The Epstein-Barr virus oncogene product, latent membrane protein 1, induces the down-regulation of E-cadherin gene expression via activation of DNA methyltransferases. Proceedings of the National Academy of Sciences. 99:10084-10089. 
Tullman MJ. 2013. Overview of the epidemiology, diagnosis and disease progression associated with multiple sclerosis. American Journal of Managed Care. 19:15-20.

Wan ES, Weiliang Q, Baccarelli A, Carey VJ, Bacherman H, Rennard SI, Agusti A, Anderson W, Lomas DA, DeMeo DL. 2012. Cigarette smoking behaviours and time since quitting are associated with differential DNA methylation across the human genome. Human Molecular Genetics. 21:3073-3082.

Wei Q, Miskimins WK, Miskimins R. 2005. Stage-specific expression of myelin basic protein in oligodendrocytes involves $\mathrm{Nkx}$ 2.2-mediated repression that is relieved by the Spl transcription factor. Advances in Experimental Medicine and Biology. 280:16284-16294.
Willer CJ, Dyment DA, Sadovnick AD, Murray TJ, Ebers GC. 2005. Timing of birth and risk of multiple sclerosis: population based study. British Medical Journal. 330:120.

Zhao Q, Rank G, Tan YT, Li H, Moritz RL, Simpson RJ, Cerruti L, Curtis DJ, Patel DJ, Allis CD, et al. 2009. PRMT5-mediated methylation of histone H4R3 recruits DNMT3A, coupling histone and DNA methylation in gene silencing. Nature Structural and Molecular Biology. 16:304-311.

Zhou Y, Simpson RJ, Holloway AF, Charlesworth J, van der Mei I, Taylor BV. 2014. The potential role of epigenetic modifications in the heritability of multiple sclerosis. Multiple Sclerosis Journal. 20:135-140. 